\title{
Avaliação clínica comparativa de duas técnicas no tratamento artroscópico das lesões parciais articulares do manguito rotador*
}

\section{Clinical Evaluation of the Arthroscopic Treatment of Partial Articular Rotator Cuff Tears}

\author{
Guilherme do Val Sella ${ }^{10}$ Luciana Andrade da Silva ${ }^{1}$ Caio Santos Checchia ${ }^{1}$ \\ Hector Carmona Marmille ${ }^{1}$ Cláudio Santili ${ }^{2} \quad$ Alberto Naoki Miyazaki ${ }^{1}$
}

1 Grupo de Cirurgia de Ombro e Cotovelo, Departamento de Ortopedia e Traumatologia, Faculdade de Ciências Médicas da Santa Casa de São Paulo, São Paulo, SP, Brasil

2 Departamento de Ortopedia e Traumatologia, Faculdade de Ciências Médicas da Santa Casa de São Paulo, São Paulo, SP, Brasil

\begin{abstract}
Endereço para correspondência Luciana Andrade da Silva, Dra., Rua Estuário, 519 - Chácara Monte Alegre, São Paulo, SP, 04645-100, Brasil (e-mail: lucalu@terra.com.br).
\end{abstract}

Rev Bras Ortop 2021;56(6):726-732.

\begin{abstract}
Resumo
Palavras-chave

- manguito rotador

- artroscopia

- tratamento

Objetivos Avaliar os resultados clínicos dos pacientes submetidos ao tratamento artroscópico da lesão parcial da porção articular do manguito rotador pelas técnicas de sutura transtendão e após completada a lesão e comparar o tempo de recuperação pósoperatório das duas técnicas.

Método Estudo retrospectivo baseado na identificação de todos os casos com lesão parcial da porção articular do manguito rotador submetidos a tratamento artroscópico no período de outubro de 1999 a dezembro de 2016 pelo Grupo de Ombro e Cotovelo da nossa instituição. Foram incluídos 39 pacientes divididos em 2 grupos: os que foram submetidos à técnica transtendínea e os outros em que a lesão foi completada. Os dois grupos foram estatisticamente semelhantes. Foi realizada a análise dos respectivos prontuários, da identificação da técnica aplicada, dos resultados pós-operatórios e das complicações relacionadas. A avaliação funcional foi realizada através do escore da University of California at Los Angeles (UCLA).

Resultados Não houve diferença estatisticamente relevante entre os grupos, com escore UCLA médio de 32, não havendo diferença também no tempo necessário para reabilitação.
\end{abstract}

\footnotetext{
Trabalho realizado no Grupo de Cirurgia de Ombro e Cotovelo do Departamento de Ortopedia e Traumatologia da Faculdade de Ciências Médicas da Santa Casa de São Paulo, "Pavilhão Fernandinho Simonsen" (DOT - FCMSCSP) (Diretor: Professora Doutora Maria Fernanda Silber Caffaro), São Paulo/SP, Brasil.
}

recebido

06 de Junho de 2019

aceito

01 de Dezembro de 2020
DOI https://doi.org/ $10.1055 / \mathrm{s}-0041-1729583$ ISSN 0102-3616.
(C) 2021. Sociedade Brasileira de Ortopedia e Traumatologia. All rights reserved.

This is an open access article published by Thieme under the terms of the Creative Commons Attribution-NonDerivative-NonCommercial-License, permitting copying and reproduction so long as the original work is given appropriate credit. Contents may not be used for commercial purposes, or adapted, remixed, transformed or built upon. (https://creativecommons.org/ licenses/by-nc-nd/4.0/)

Thieme Revinter Publicações Ltda., Rua do Matoso 170, Rio de Janeiro, RJ, CEP 20270-135, Brazil 


\begin{abstract}

\section{Keywords}

- rotator cuff

- arthroscopy

- treatment

Objectives To evaluate the clinical results of patients submitted to arthroscopic treatment of partial lesion of the articular part of the rotator cuff by transtendon suture techniques and after completing the lesion and to compare the postoperative recovery time of the two techniques.

Method Retrospective study based on the identification of all cases with partial lesion of the articular part of the rotator cuff submitted to arthroscopic treatment from October 1999 to December 2016 at the Shoulder and Elbow Group of our institution. Thirty-nine patients were included divided into two groups: those who underwent the transtendon technique and those in whom the lesion was completed. The two groups were statistically similar. The respective medical records were analyzed as well as the identification of the applied technique, the postoperative results, and the related complications. The functional evaluation was performed using the score of the University of California at Los Angeles (UCLA).

Results There was no statistically relevant difference between the groups, with a mean UCLA score of 32 , and no difference in the time required for rehabilitation.

Conclusion There was no difference between the clinical outcome of the patients, regardless of the technique used to repair the partial lesions of the articular part of the rotator cuff with satisfactory results in $93 \%$ of the cases.
\end{abstract}

Conclusão Não houve diferença entre o resultado clínico dos pacientes, independente da técnica empregada para o reparo das lesões parciais da porção articular do manguito rotador com resultados satisfatórios em $93 \%$ dos casos.

\section{Introdução}

A lesão parcial do manguito rotador é reconhecida como uma importante causa de disfunção e dor no ombro. ${ }^{1}$ Sua incidência ainda não é consenso na literatura, apesar de existirem diversos estudos sobre ela. ${ }^{2-4}$ Sher et al., ${ }^{3}$ analisando 96 ombros assintomáticos através de ultrassonografia, encontraram $20 \%$ de lesões parciais. Ao dissecar 249 ombros de cadáveres, Lohr e Uhthoff $^{2}$ encontraram $32 \%$ de lesões parciais, enquanto Fukuda $^{5}$ encontrou 13\%. Esses autores descreveram que cerca de $1 / 3(27 \%)$ dessas lesões acometem a porção articular.

Ellman ${ }^{6}$ foi o primeiro a propor, baseado em achados artroscópicos, um sistema de classificação da lesão parcial em relação a sua localização (A, articular; B, bursal; C, intratendínea) e a sua extensão (grau 1: < $3 \mathrm{~mm}$; grau 2: de 3-6 mm; grau 3: > $6 \mathrm{~mm}$ ).

Quanto à fisiopatologia das lesões parciais da porção articular (LPPAs) do manguito rotador, acredita-se que elas podem ocorrer tanto com mecanismos intrínsecos (área de hipovascularização e alterações metabólicas relativas à idade) quanto extrínsecos (impacto interno póstero-superior, eventos traumáticos agudos e microtraumas de repetição), ou até mesmo uma combinação entre eles. ${ }^{7}$ Weinreb et al., ${ }^{8}$ em 2014, publicaram um artigo mostrando que existem evidências de que fatores intrínsecos relacionados ao tendão levam à degeneração de suas fibras na face articular com afilamento e desorientação dessas, resultando na lesão anatômica dessa região. Corroborando essas evidências, Ozaki et al. ${ }^{9}$ não encontraram sinais de impacto subacromial nas lesões articulares do manguito rotador e, portanto, a excluem como possível causa para essas lesões. Outro mecanismo que leva à LPPA do manguito rotador é encontrado nos atletas arremessadores, que podem ter um impacto interno póstero-superior. ${ }^{4,10}$

O tratamento inicial da LPPA do manguito rotador é realizado com medidas conservadoras, tais como fisioterapia e mudança dos hábitos para evitar a progressão da lesão. ${ }^{11} \mathrm{O}$ tratamento cirúrgico está indicado na falha do tratamento conservador e nos casos em que a lesão acomete mais de 50\% da espessura do tendão, devido a um risco maior de progressão da lesão; 40\% das LPPAs evoluem para lesões completas, de acordo com Mall et al. ${ }^{12}$ e Stuart et al. ${ }^{13}$

Diferentes técnicas cirúrgicas artroscópicas têm sido descritas para abordagem desse tipo de lesão, desde um simples desbridamento artroscópico do tendão, acreditando na sua capacidade de cicatrização (desde que realizado em lesões de baixo grau ${ }^{14}$ ), à realização de um reparo transtendão da lesão, descrito inicialmente por Stephen Snyder, ${ }^{15}$ ou ainda a desinserção da área de tendão acometido ("completando a lesão") seguida do seu reparo. ${ }^{16}$

O objetivo principal deste trabalho é avaliar os resultados clínicos dos pacientes submetidos ao tratamento artroscópico das LPPAs pelas técnicas de sutura transtendínea e após completada a lesão e comparar o tempo de recuperação pósoperatório das duas técnicas.

\section{Casuísticas e métodos}

No período de outubro de 1999 a dezembro de 2016, 39 pacientes com diagnóstico de LPPA foram submetidos a tratamento cirúrgico artroscópico realizado pelo Grupo de 
Ombro e Cotovelo do Departamento de Ortopedia e Traumatologia da nossa instituição.

Os pacientes, todos com lesão parcial articular do tendão do músculo supra-espinal, foram divididos em dois grupos: grupo I, os que foram submetidos ao reparo transtendão, e o grupo II, pacientes submetidos ao tratamento com reparo após transformar a lesão parcial em completa.

Os critérios de inclusão foram: pacientes com diagnóstico de lesão parcial da porção articular do manguito rotador, realizado por meio de imagens de ressonância magnética do período pré-operatório, submetidos a tratamento artroscópico, e que tiveram seguimento mínimo $\geq 2$ anos.

No grupo I, com 19 pacientes e média de seguimento de 4,61 anos, 13 eram do sexo masculino (68\%) e 6 do sexo feminino. A média de idade foi de 48 anos, com variação de 34 a 70 anos. 0 lado dominante foi acometido em $73 \%$ dos casos; 16 pacientes (84\%) praticavam esporte e 9 pacientes (47\%) relataram evento traumático como desencadeante do quadro.

No grupo II, com 20 pacientes e média de seguimento de 4,35 anos, 11 eram do sexo masculino (55\%) e 9 do sexo feminino. A média de idade foi de 52 anos, com variação de 29 a 75 anos. $O$ lado dominante foi acometido em $85 \%$ dos casos, 11 pacientes (55\%) praticavam esporte e apenas 3 pacientes relataram evento traumático.

Não houve diferenças estatisticamente significantes entre as amostras em relação a idade, sexo e membro acometido, sendo que em $78 \%$ dos casos o lado dominante foi afetado. Todos os pacientes foram submetidos ao procedimento cirúrgico em posição de cadeira de praia, sob anestesia geral associada a bloqueio anestésico do plexo braquial. Foi realizada inspeção artroscópica articular, seguida de inspeção subacromial e bursectomia parcial. No grupo I, sete pacientes apresentavam lesão do tipo SLAP, dois com artrose da articulação acromioclavicular, um com fratura do tubérculo maior, já consolidada e que foi tratada conservadoramente, um paciente com lesão do cabeça longa do bíceps e um paciente com lesão de Bankart. Já no grupo II, seis pacientes apresentavam lesão do tipo superior labral anterior posterior (SLAP), quatro com ruptura do cabeça longa do bíceps e três com artrose da articulação acromioclavicular. A partir desse ponto, as técnicas se diferenciam: nos pacientes do grupo I foi realizada a passagem dos fios das âncoras via transtendão, através do espaço articular para a superfície bursal, seguindo com o reparo. Quando optamos por esse tipo de reparo, aumentamos a pressão da bomba de infusão de soro para perto de $80 \mathrm{mmHg}$, o que faz com que o espaço articular aumente e o tendão levante do seu leito de inserção e possibilite a visualização intra-articular da passagem das âncoras de subacromial para intra-articular e seu correto posicionamento no chamado footprint. Depois de fixada a âncora, um fio é pego com uma pinça tipo birdbick e passado através do tendão num ponto de tecido considerado sadio, isto é, fora da área da lesão. Retornamos a pressão da bomba para o valor normal, para evitar um edema exagerado e rápido que impeça o restante da cirurgia. Depois, a ótica é posicionada novamente no espaço subacromial e os fios são amarrados com pontos simples de correr. O procedimento é repetido quantas vezes se achar necessário (-Figura 1). Já nos pacientes do grupo II, foi realizada a desinserção das fibras bursais que se

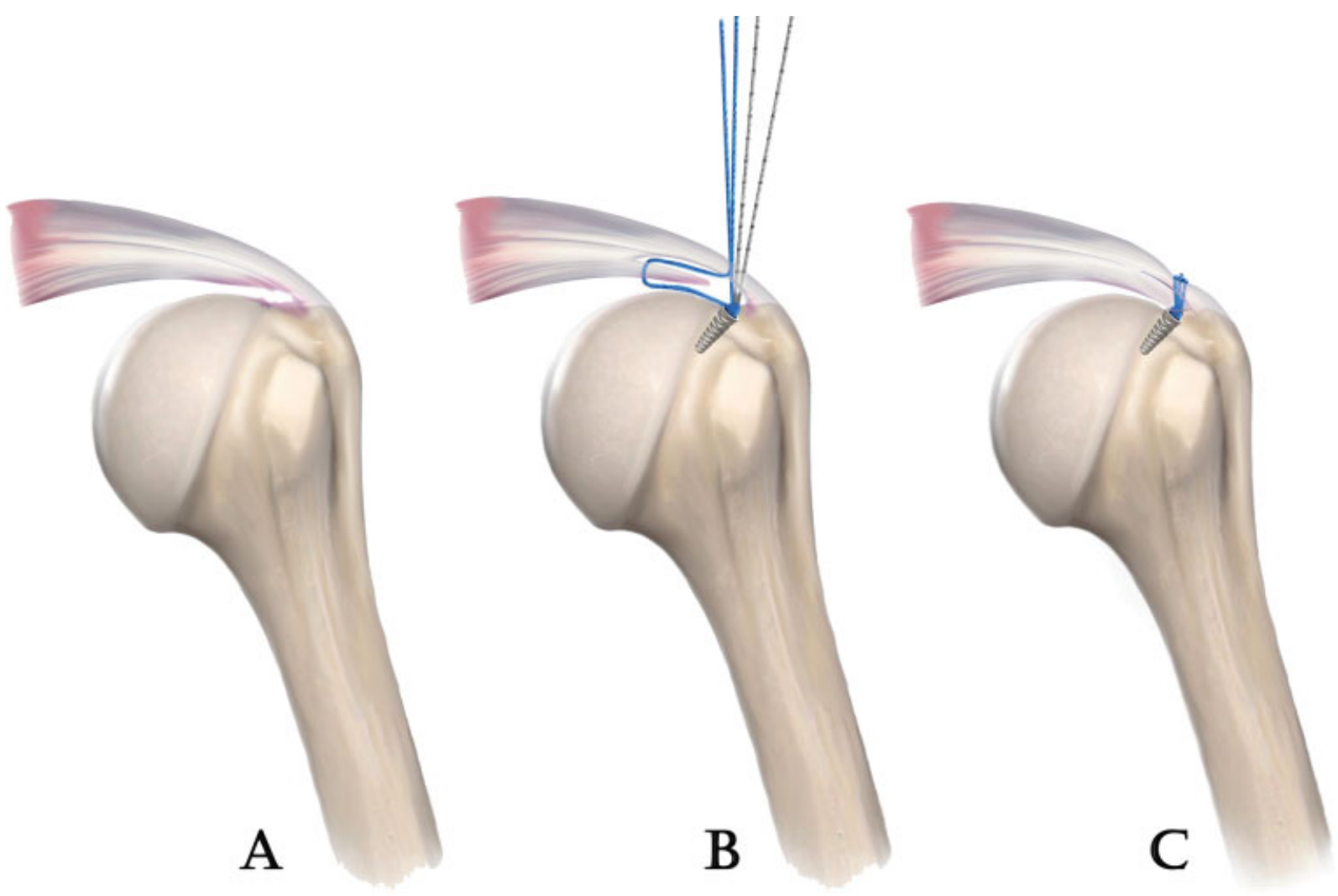

Fig. 1 Reparo transtendão da lesão parcial articular do tendão do músculo supraespinal. A - Representação da lesão parcial articular, B - Forma de passagem dos fios de sutura de acordo com esta técnica, C - Lesão suturada. 


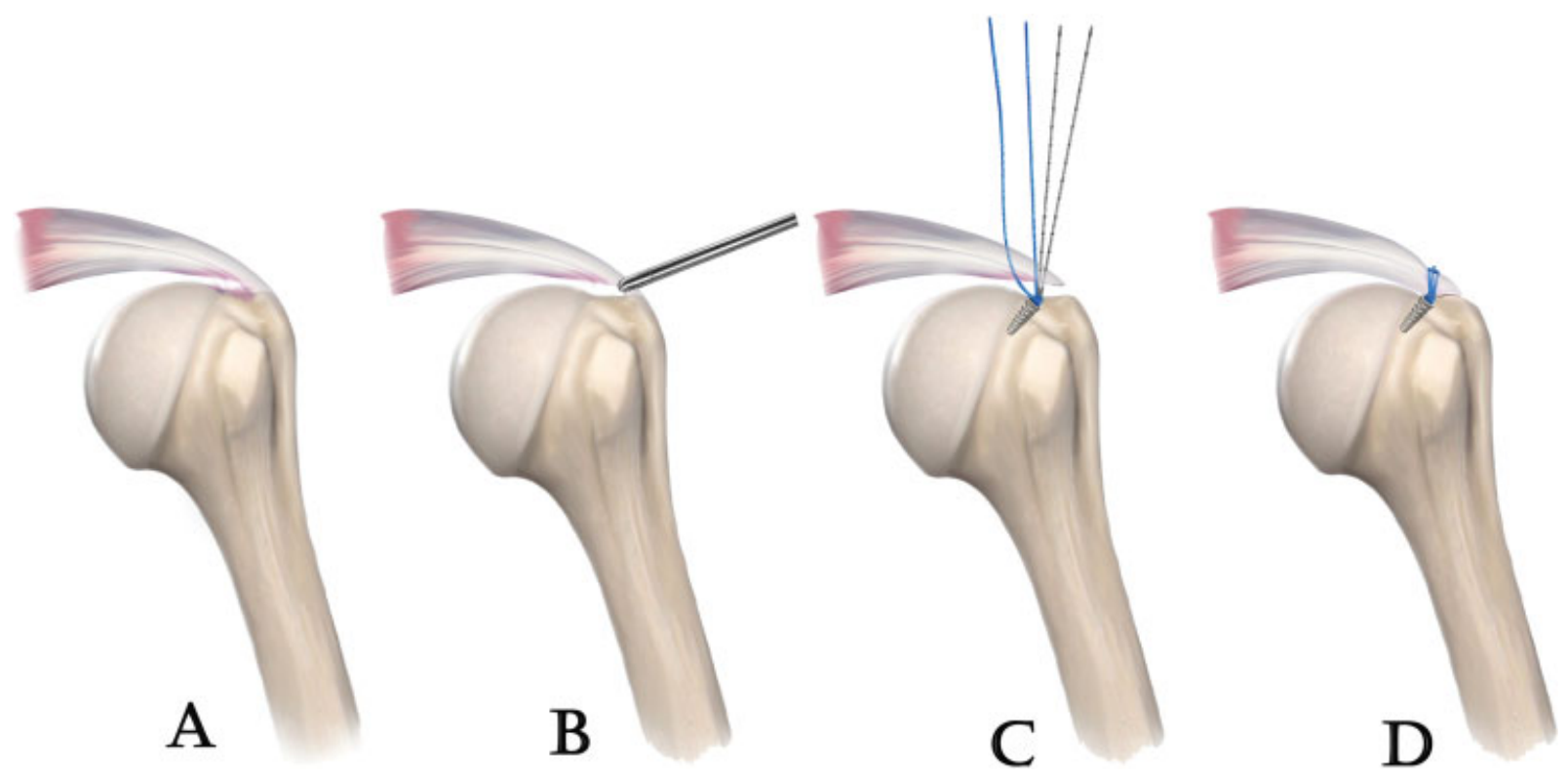

Fig. 2 Reparo após transformar a lesão parcial em completa na lesão parcial articular do tendão do músculo supraespinal. A - Representação da lesão parcial articular, B - Lesão parcial sendo completada, C-Forma de passagem dos fios de sutura na lesão já completada, D - Lesão suturada.

mantinham intactas, transformando-a em uma lesão completa, sendo seguido do reparo por meio da passagem dos fios de sutura pela face bursal (-Figura 2). Todas as lesões foram reparadas pela técnica de pontos simples via artroscópica.

No período pós-operatório, os pacientes ficaram imobilizados com tipoia funcional entre 4 e 6 semanas, dando sequência à reabilitação.

Foi avaliada a amplitude do arco de movimento aferindo-se a elevação, rotações lateral e medial. A avaliação clínica foi realizada por meio do escore da University of California at Los Angeles (UCLA). ${ }^{17}$ Para a avaliação do tempo de recuperação, utilizamos a comparação do tempo que os pacientes dos dois grupos demoraram para atingir o seu valor "máximo" do escore do UCLA, ${ }^{17}$ por teste estatístico específico listado a seguir.

Inicialmente foi realizada análise descritiva com cálculo da distribuição de porcentagens para cada variável. As variáveis qualitativas (sexo, dominância, esporte, capsulite intra e pósoperatória) foram analisadas pelo teste de independência Quiquadrado, quando respeitadas as hipóteses de independência da amostra, e pelo teste exato de Fisher quando não respeitada. Já as variáveis quantitativas e qualitativas ordinais (elevação, rotações medial e lateral, pré e pós-operatória, tempo de seguimento, valor do UCLA e tempo do UCLA) foram analisadas pelo teste não paramétrico de Mann-Withney, quando a distribuição não aderiu à distribuição normal, e pelo teste $F$, quando houve aderência à distribuição normal, seguido pela análise da igualdade das médias pelo teste T-de-Student.

As análises foram realizadas utilizando-se o programa estatístico Minitab v.17 (Minitab, LLC, State College, PA, EUA),. Foram rejeitadas todas as hipóteses com níveis descritivos (valor de $p$ ) inferiores a 0,05 , que foi o nível de significância adotado.

O trabalho foi submetido ao Comitê de Ética em Pesquisa e aprovado conforme CAAE: 79428617.2.0000.5479.

\section{Resultados}

Utilizando o escore do UCLA, ${ }^{17}$ no grupo I tivemos nove resultados excelentes (42\%), 8 resultados bons (38\%) e 2 resultados insatisfatórios (-Tabela 1). A média de tempo para reabilitação foi de 6 meses, arco de movimento pósoperatório com elevação média de $142^{\circ}\left(110-160^{\circ}\right)$, rotação lateral de $59^{\circ}\left(45-70^{\circ}\right)$ e rotação medial de T8 (T3-L5).

Já no grupo II, tivemos 5 resultados excelentes (25\%), 14 resultados bons (70\%) e 1 resultado insatisfatório (- Tabela 2 ). A média de tempo para reabilitação foi de 5,2 meses, arco de movimento pós-operatório com elevação média de $146^{\circ}$ $\left(130-160^{\circ}\right)$, rotação lateral de $56^{\circ}\left(45-70^{\circ}\right)$ e rotação medial de T8 (T5-L2).

Como complicação tivemos dois casos de capsulite adesiva, sendo um em cada grupo. O paciente do grupo I foi submetido à liberação artroscópica após 8 meses do reparo da lesão atingindo o escore UCLA ${ }^{17}$ de 33 após 1 ano. 0 paciente do grupo II apresentou melhora após a realização de bloqueios do nervo supra-escapular e fisioterapia, atingindo o escore UCLA ${ }^{17}$ de 33 após 7 meses de pós-operatório. ${ }^{18}$

Após análise estatística, não houve diferença entre o UCLA ${ }^{16}$ médio para ambos os grupos, com resultado final de 32 , variando para o grupo I de 22 a 35 e o grupo II de 23 a 35 . Na análise do tempo necessário para atingir o resultado final do escore UCLA, ${ }^{17}$ houve uma diferença de 3 semanas na média, porém sem relevância estatística, com valor de $p=0,336$.

\section{Discussão}

O tratamento cirúrgico das LPPAs está indicado na falha do tratamento conservador e comumente nos casos em que a lesão acomete mais de $50 \%$ das fibras do tendão, ${ }^{13}$ devido a um risco de $40 \%$ na progressão para lesão completa, conforme descrevem Mall et al. ${ }^{12}$ Mazzoca et al. $^{19}$ também 
Tabela 1 Dados clinicos dos pacientes submetidos a reparo transtendão

\begin{tabular}{|c|c|c|c|c|c|c|c|}
\hline $\begin{array}{l}\mathrm{N}^{\circ} \text { de } \\
\text { pacientes }\end{array}$ & Sexo & Idade & $\begin{array}{l}\text { Lado } \\
\text { dominante }\end{array}$ & $\Delta \mathrm{tt}$ & UCLA & $\Delta \mathrm{t}$ & COMPLICAÇÕES \\
\hline 1 & $\mathrm{~F}$ & 50 & & $03 \mathrm{a}$. & TOTAL:35 & $4 \mathrm{~m}$ & \\
\hline 2 & $M$ & 70 & & 02 а. & TOTAL: 35 & $5 \mathrm{~m}$ & \\
\hline 3 & $\mathrm{~F}$ & 49 & + & 03 а. & TOTAL: 35 & $5 \mathrm{~m}$ & \\
\hline 4 & $\mathrm{~F}$ & 39 & + & 02 a. & TOTAL: 35 & $5 \mathrm{~m}$ & \\
\hline 5 & $\mathrm{M}$ & 51 & + & 03 a. & TOTAL: 35 & $4 m$ & \\
\hline 6 & $\mathrm{M}$ & 43 & + & 07 a. & TOTAL: 22 & $9 \mathrm{~m}$ & \\
\hline 7 & $M$ & 49 & + & 02 a. & TOTAL: 35 & $4 m$ & \\
\hline 8 & $M$ & 40 & + & 09 a. & TOTAL: 32 & $6 \mathrm{~m}$ & \\
\hline 9 & $M$ & 34 & + & 2 a. & TOTAL: 35 & $5 \mathrm{~m}$ & \\
\hline 10 & M & 44 & + & 09 a. & TOTAL: 32 & $4 \mathrm{~m}$ & \\
\hline 11 & $\mathrm{~F}$ & 56 & & 09 a. & TOTAL: 31 & $5 \mathrm{~m}$ & \\
\hline 12 & $M$ & 41 & + & 12 a. & TOTAL: 34 & $4 m$ & \\
\hline 13 & $\mathrm{~F}$ & 54 & + & 02 a. & TOTAL: 32 & $9 \mathrm{~m}$ & \\
\hline 14 & M & 52 & + & 09 a. & TOTAL: 31 & $8 \mathrm{~m}$ & \\
\hline 15 & $M$ & 58 & & 04 a. & TOTAL: 23 & $10 \mathrm{~m}$ & \\
\hline 16 & $\mathrm{M}$ & 59 & + & 09 a. & TOTAL: 34 & $5 \mathrm{~m}$ & \\
\hline 17 & $\mathrm{~F}$ & 53 & & 05 a. & TOTAL: 33 & $6 \mathrm{~m}$ & \\
\hline 18 & M & 44 & + & 05 a. & TOTAL: 33 & $4 m$ & \\
\hline 19 & $M$ & 35 & + & 07 a. & TOTAL: 33 & $12 \mathrm{~m}$ & $\begin{array}{l}\text { REVISÃO PARA LIBERAÇÃO COM } \\
8 \text { MESES DEVIDO CAPSULITE }\end{array}$ \\
\hline
\end{tabular}

Abreviaturas: a: anos; F: feminino; m: meses; M: masculino; $\Delta$ t: tempo para reabilitação; $\Delta$ tt: tempo de seguimento pós-operatório; UCLA, University of California at Los Angeles.

demonstraram que nos casos em que há mais de 50\% de lesão das fibras do tendão, o tendão remanescente apresenta um aumento de sua tensão, o que levaria a sua eventual ruptura completa.

No ato operatório, ao nos deparamos com LPPA, pode-se abordá-la de duas maneiras em relação ao tendão: ou mantendo sua integridade na inserção - técnica conhecida como fixação transtendão da lesão - ou por uma segunda técnica na qual há a desinserção completa das fibras remanescentes, transformando-a numa lesão completa.

Estudos como de Sethi et al. ${ }^{20}$ e Mihata et al. ${ }^{21}$ mostram vantagens biomecânicas do reparo transtendão. Nesta técnica, as fibras bursais (intactas e sem lesão) e articulares (contendo a lesão) são suturadas separadamente (- Figura 1), o que proporciona menor tensão ao final da sutura nas fibras bursais, além de uma maior área de contato na área de inserção. Mas conforme observado por Shin, ${ }^{22}$ esses pacientes apresentam um pós-operatório mais doloroso e com uma recuperação do arco de movimento mais lenta, o que poderia estar relacionado a lesões com grande retração da parte articular acometida, ocorrendo um desbalanço entre as fibras reparadas e as que foram mantidas íntegras. Em nosso estudo, observamos que os pacientes do grupo I demonstram um mês a mais na média do tempo de recuperação para atingir o escore UCLA ${ }^{17}$ final do que os pacientes do grupo II, mas essa diferença não se mostrou estatisticamente significante.
Shin ${ }^{22}$ cita também que a desinserção de toda a margem lateral do manguito rotador, ao transformar a lesão parcial em completa, pode levar ao risco do reparo não anatômico, alterando a biomecânica e assim acarretando uma degeneração precoce do tendão. Porém, em nossa opinião, assim como na de Shin, ${ }^{22}$ tal manobra possibilita uma melhor visão e facilita o reparo devido à maior familiaridade com a técnica. ${ }^{11,20}$ Apesar do bom resultado funcional não ser um fator especifico para sabermos se houve ou não reparo anatômico da lesão, não suspeitamos de nenhuma alteração biomecânica importante, visto que os pacientes do grupo II apresentaram boa evolução pós-operatória, com retorno das atividades habituais, assim como encontrado no estudo de Godinho et al., ${ }^{23}$ que obtiveram $100 \%$ de satisfação e resultados bons/excelentes com a técnica.

Não há consenso na literatura sobre qual a melhor técnica no reparo desse tipo de lesão. Os trabalhos comparativos entre as duas técnicas, como os de Shin, ${ }^{22}$ Ono et al. ${ }^{24}$ e Castagna et al., ${ }^{16}$ não apresentam diferenças estatísticas entre elas, apresentando resultados semelhantes no pós-operatório, com a mesma taxa de cicatrização, re-rupturas, e resultados finais. Nosso estudo demonstra que ambas as técnicas possibilitam bons resultados finais, sem diferenças estatísticas ao avaliar o escore UCLA ${ }^{17}$ entre os dois grupos, com valor de $p=0,321$, inclusive com tempo de reabilitação pós-operatória semelhante, com valor de $p=0,336$. 
Tabela 2 Dados clínicos dos pacientes submetidos a reparo após completada a lesão

\begin{tabular}{|c|c|c|c|c|c|c|c|}
\hline Caso & Sexo & Idade & Lado dominante & $\Delta \mathrm{tt}$ & UCLA & $\Delta \mathrm{t}$ & COMPLICAÇÕES \\
\hline 1 & $\mathrm{M}$ & 58 & + & 02 a. & TOTAL: 32 & $6 \mathrm{~m}$ & \\
\hline 2 & $\mathrm{~F}$ & 63 & + & 02 a, $10 \mathrm{~m}$ & TOTAL: 31 & $5 \mathrm{~m}$ & \\
\hline 3 & $\mathrm{~F}$ & 65 & + & 02 a. & TOTAL: 32 & $4 \mathrm{~m}$ & \\
\hline 4 & $\mathrm{~F}$ & 67 & + & 02 a. & TOTAL: 33 & $5 \mathrm{~m}$ & \\
\hline 5 & $M$ & 75 & & 03 a. & TOTAL: 34 & $4 \mathrm{~m}$ & \\
\hline 6 & $\mathrm{~F}$ & 57 & & 05 a. & TOTAL: 34 & $6 \mathrm{~m}$ & \\
\hline 7 & $\mathrm{M}$ & 39 & + & 08 a. & TOTAL: 32 & $6 \mathrm{~m}$ & \\
\hline 8 & $\bar{F}$ & 73 & + & 05 a. & TOTAL: 35 & $5 \mathrm{~m}$ & \\
\hline 9 & $\mathrm{~F}$ & 59 & + & 02 a. & TOTAL: 32 & $5 \mathrm{~m}$ & \\
\hline 10 & $\mathrm{M}$ & 43 & + & 04 a. & TOTAL: 32 & $7 \mathrm{~m}$ & \\
\hline 11 & $M$ & 38 & + & 02 a. & TOTAL: 33 & $7 \mathrm{~m}$ & \\
\hline 12 & $\mathrm{~F}$ & 29 & + & 11 a. & TOTAL: 34 & $4 \mathrm{~m}$ & \\
\hline 13 & $\mathrm{M}$ & 44 & & 13 a. & TOTAL: 23 & $6 \mathrm{~m}$ & \\
\hline 14 & $\mathrm{M}$ & 42 & + & 02 a. & TOTAL: 32 & $5 \mathrm{~m}$ & \\
\hline 15 & $\mathrm{~F}$ & 51 & & 08 a. & TOTAL: 33 & $4 \mathrm{~m}$ & \\
\hline 16 & $M$ & 39 & + & 06 a. & TOTAL: 32 & $4 \mathrm{~m}$ & \\
\hline 17 & $M$ & 49 & + & 02 a. & TOTAL: 33 & $4 \mathrm{~m}$ & \\
\hline 18 & $M$ & 51 & + & 03 a. & TOTAL: 32 & $5 \mathrm{~m}$ & \\
\hline 19 & $M$ & 56 & + & 03 a. & TOTAL: 33 & $7 \mathrm{~m}$ & $\begin{array}{l}\text { CAPSULITE COM } 3 \text { MESES SENDO } \\
\text { REALIZADO BLOQUEIOS E FISIOTERAPIA. }\end{array}$ \\
\hline 20 & $\bar{F}$ & 47 & + & 02 a. & TOTAL: 35 & $5 \mathrm{~m}$ & \\
\hline
\end{tabular}

Abreviaturas: a: anos; F: feminino; m: meses; M: masculino; $\Delta$ t: tempo para reabilitação; $\Delta$ tt: tempo de seguimento pós-operatório; UCLA, University of California at Los Angeles.

Apesar do bom resultado obtido com as duas técnicas, tivemos em nosso estudo três casos com resultados insatisfatórios, sendo dois do grupo I e um do II. No grupo I, o primeiro paciente tinha 43 anos, era tenista não profissional com lesão acometendo o lado dominante com sintomas por cerca de 20 anos. Com boa mobilidade pré-operatória $\left(140^{\circ}, 80^{\circ}, \mathrm{T} 3\right)$, o paciente evoluiu com uma perda no pós-operatório de $110^{\circ}$, $45^{\circ}$ e L5, que se manteve. Evoluiu com dor ao executar trabalhos leves, sendo capaz de realizar todas as atividades domésticas, mas com limitação ao realizar movimentos acima da linha do ombro (força grau IV). O segundo caso do grupo I era um paciente de 58 anos, nadador também amador, com sintomas do lado não dominante por 3 anos. Arcos de movimento pré e pós-operatório bons, sem limitação. Evoluiu também com dor ao executar trabalhos leves, com perda grau IV de força acima do ombro. Apesar de tudo, os dois se dizem satisfeitos com o resultado. Já o caso do grupo II era um paciente de 44 anos, sem histórico de prática de esporte, apresentando sintomas há 1 ano. Arco de movimento préoperatório $160^{\circ}, 70^{\circ}, \mathrm{T} 7$. Evoluiu no pós-operatório com dor ao executar trabalhos leves, com discreta diminuição da elevação e rotação lateral, sem perda de força. No entanto este paciente está insatisfeito com o resultado devido à persistência da dor. $\mathrm{O}$ que nos chamou atenção nos três casos é que não encontramos uma causa objetiva para a manutenção da dor. Todos os pacientes apresentam exame de imagem mostrando cicatrização normal da lesão.

\section{Conclusão}

Tanto a sutura transtendínea quanto a sutura após completar a lesão levaram a resultados satisfatórios em 93\% dos pacientes na nossa série. Não encontramos diferença estatística no tempo de reabilitação entre os dois grupos.

\section{Suporte Financeiro}

Não houve suporte financeiro de fontes públicas, comerciais, ou sem fins lucrativos.

Conflito de Interesses

Os autores declaram não haver conflito de interesses.

\section{Referências}

1 Stuart KD, Karzel RP, Ganjianpour M, Snyder SJ. Long-term outcome for arthroscopic repair of partial articular-sided supraspinatus tendon avulsion. Arthroscopy 2013;29(05):818-823

2 Loehr JF, Uhthoff HK. The pathogenesis of degenerative rotator cuff tears. Orthop Trans 1987;11:237-243

3 Sher JS, Uribe JW, Posada A, Murphy BJ, Zlatkin MB. Abnormal findings on magnetic resonance images of asymptomatic shoulders. J Bone Joint Surg Am 1995;77(01):10-15 
4 Hawi N, Liodakis E, Garving C, Habermeyer P, Tauber M. Pulley lesions in rotator cuff tears: prevalence, etiology, and concomitant pathologies. Arch Orthop Trauma Surg 2017;137(08): 1097-1105

5 Fukuda H. Partial-thickness rotator cuff tears: a modern view on Codman's classic. J Shoulder Elbow Surg 2000;9(02):163-168

6 Ellman H. Diagnosis and treatment of incomplete rotator cuff tears. Clin Orthop Relat Res 1990;(254):64-74

7 Finnan RP, Crosby LA. Partial-thickness rotator cuff tears. J Shoulder Elbow Surg 2010;19(04):609-616

8 Weinreb JH, Sheth C, Apostolakos J, et al. Tendon structure, disease, and imaging. Muscles Ligaments Tendons J 2014;4(01): 66-73

9 Ozaki J, Fujimoto S, Nakagawa Y, Masuhara K, Tamai S. Tears of the rotator cuff of the shoulder associated with pathological changes in the acromion. A study in cadavera. J Bone Joint Surg Am 1988; 70(08):1224-1230

10 Carvalho CD, Cohen C, Belangero PS, et al. Lesão parcial do manguito rotador no atleta - bursal ou articular? Rev Bras Ortop 2015;50(04):416-421

11 Kim YS, Lee HJ, Bae SH, Jin H, Song HS. Outcome comparison between in situ repair versus tear completion repair for partial thickness rotator cuff tears. Arthroscopy 2015;31(11):2191-2198

12 Mall NA, Kim HM, Keener JD, et al. Symptomatic progression of asymptomatic rotator cuff tears: a prospective study of clinical and sonographic variables. J Bone Joint Surg Am 2010;92(16): 2623-2633

13 Stuart KD, Karzel RP, Ganjianpour M, Snyder SJ. Long-term outcome for arthroscopic repair of partial articular-sided supraspinatus tendon avulsion. Arthroscopy 2013;29(05):818-823

14 Veado MAC, Bertolini FM, Almeida Filho IA. Eficácia do desbridamento artroscópico nas lesões parciais do manguito rotador. Rev Bras Ortop 2006;41(1/2):22-28
15 Snyder SJ. Arthroscopic repair of partial articular supraespinatus tendo avulsion: Pasta lesion of the rotator cuff tendon. In: Shoulder arthoscopy. Philadelphia: Lippincott Williams \& Wilkins; 2003:219-229

16 Castagna A, Borroni M, Garofalo R, et al. Deep partial rotator cuff tear: transtendon repair or tear completion and repair? A randomized clinical trial. Knee Surg Sports Traumatol Arthrosc 2015;23 (02):460-463

17 Ellman H, Hanker G, Bayer M. Repair of the rotator cuff. End-result study of factors influencing reconstruction. J Bone Joint Surg Am 1986;68(08):1136-1144

18 Checchia SL, Fregoneze M, Miyazaki AN, et al. Tratamento da capsulite adesiva com bloqueios seriados do nervo supra-escapular. Rev Bras Ortop 2006;41(07):245-252

19 Mazzocca AD, Rincon LM, O'Connor RW, et al. Intra-articular partial-thickness rotator cuff tears: analysis of injured and repaired strain behavior. Am J Sports Med 2008;36(01):110-116

20 Sethi PM, Rajaram A, Obopilwe E, Mazzocca AD. Partial articularsided rotator cuff tears: in situ repair versus tear completion prior to repair. Orthopedics 2013;36(06):771-777

21 Mihata T, McGarry MH, Ishihara Y, et al. Biomechanical analysis of articular-sided partial-thickness rotator cuff tear and repair. Am J Sports Med 2015;43(02):439-446

22 Shin SJ. A comparison of 2 repair techniques for partial-thickness articular-sided rotator cuff tears. Arthroscopy 2012;28(01): 25-33

23 Godinho GG, França FO, Freitas JMA, et al. Avaliação funcional a longo prazo do tratamento videoartroscópico das lesões parciais do manguito rotador. Rev Bras Ortop 2015;50(02):200-205

24 Ono Y, Woodmass JM, Bois AJ, Boorman RS, Thornton GM, Lo IK. Arthroscopic Repair of Articular Surface Partial-Thickness Rotator Cuff Tears: Transtendon Technique versus Repair after Completion of the Tear-A Meta-Analysis. Adv Orthop 2016;2016:7468054 